\title{
Evolutionary Spectrum for Random Field and Missing Observations
}

\author{
Rachid Sabre \\ AgroSup/Laboratoire Le2i Université de Bourgogne, 26, \\ bd Docteur Petitjean 21000 Dijon \\ r.sabre@agrosupdijon.fr
}

\begin{abstract}
There are innumerable situations where the data observed from a non-stationary random field are collected with missing values. In this work a consistent estimate of the evolutionary spectral density is given where some observations are randomly missing.
\end{abstract}

Keywords: spectral density, non-stationary processes, periodogram, smoothing estimate, oscillatory process.

\section{Introduction}

Spectral analysis for stationary processes has been extensively studied in recent years. However, in many applications the signals must be modeled as nonstationary processes. This has motivated several authors to study non-stationary processes assuming that they are locally stationary. Priestley ([14, [15]) established the theory of the evolutionary spectrum generalizing spectral analysis for stationary processes. The evolutionary spectrum is time-dependent and describe the local power-frequency distribution at each instant of time. Other studies based on the Wold-Cramér decomposition have contributed to the development of the evolutionary spectrum [10, 17], [16], 18. The applications of the evolutionary spectrum cover various scientific fields: signal and image processing [3], 1] , seismic 20], oceanography, music [4. The estimation of the evolutionary spectral density is studied in [15], [10, 8], 19, 9].

On the other hand, Jones [6] is the first to consider the missing data problems in spectral analysis. More precisely he studied the case where a block of observations is periodically unobtainable. In parallel, the theory of amplitudemodulated stationary processes was developed by Parzen [12, he applied this theory to solve periodic missing data problems. Bloomfield 2 has considered stationary processes with randomly missing data. He gives an asymptotically unbiased estimator of the spectral density and shows under suitable conditions that its variance converges to zero. We cite in this paper a few works that have contributed to find solutions to problems of missing observations: [21, [13, [7].

The aim of the present paper is to consider the problem of the randomly missing data for the class of non-stationary oscillatory random fields. Using the same techniques introduced by Bloomfield [2] for stationary processes, we give a 
consistent estimate of the evolutionary spectral density. The paper is organized as follows. In section 2, we give some notations, assumptions and the amplitude modulating function $Y_{t_{1}, t_{2}}$. In section 3 , we construct a periodogram and we show that it is an asymptotically unbiased estimator. Since, we smooth the periodogram in the neighborhood of the time-instant $t$ via a weight function and we show that it is a consistent estimate of the (weighted) average value of $h_{t_{1}, t_{2}}\left(\omega_{01}, \omega_{02}\right)$ in the neighborhood of the time-instant $\left(t_{1}, t_{2}\right)$. Section 4 is reserved to prove the theorems. In section 5 , we study numerical results and simulation. Concluding comments are given in section 6 .

\section{The Amplitude Modulating Function, $\boldsymbol{Y}_{t_{1}, t_{2}}$}

As in Priestley (14, 15]), we consider a non-stationary centred oscillatory random field $X_{t_{1}, t_{2}}, t_{1}, t_{2} \in \mathbb{Z}$ i.e.

$$
X_{t_{1}, t_{2}}=\int_{-\pi}^{+\pi} \int_{-\pi}^{+\pi} e^{i\left(t_{1} \omega_{1}, t_{2} \omega_{2}\right)} A_{t_{1}, t_{2}}\left(\omega_{1}, \omega_{2}\right) d Z_{1}\left(\omega_{1}, \omega_{2}\right) ; \quad t_{1}, t_{2} \in \mathbb{Z},
$$

where the function $A_{t_{1}, t_{2}}\left(\omega_{1}, \omega_{2}\right)$ is given by

$$
A_{t_{1}, t_{2}}\left(\omega_{1}, \omega_{2}\right)=\int_{-\infty}^{+\infty} \int_{-\infty}^{+\infty} e^{i\left(\theta_{1} t_{1}, \theta_{2} t_{2}\right)} d F_{\omega_{1}, \omega_{2}}\left(\theta_{1}, \theta_{2}\right)
$$

$t_{1}, t_{2} \in \mathbb{Z}$ and $\omega_{1}, \omega_{2} \in[-\pi, \pi]$

where $F_{\omega_{1}, \omega_{2}}$ is a measure satisfying: $\int_{-\infty}^{+\infty} \int_{-\infty}^{+\infty}\left|d F_{\omega_{1}, \omega_{2}}\left(\theta_{1}, \theta_{2}\right)\right|=1$ and $Z_{1}$ is a processus with orthogonal increments defined on the interval $[-\pi,+\pi]^{2}$ and $E\left|d Z_{1}\left(\omega_{1}, \omega_{2}\right)\right|^{2}=d \mu_{1}\left(\omega_{1}, \omega_{2}\right)$ where $\mu_{1}$ is a positive measure. The evolutionary spectral measure is defined by Priestley (14, [15]) at each $\left(t_{1}, t_{2}\right)$ by

$$
d H_{t_{1}, t_{2}}\left(\omega_{1}, \omega_{2}\right)=\left|A_{t_{1}, t_{2}}\left(\omega_{1}, \omega_{2}\right)\right|^{2} d \mu(\omega) .
$$

Our choice of oscillatory random field is motivated by the fact that it has a physical interpretation and the variance of the process is interpreted as a measure of the total power of the process at time $t$, because $\operatorname{Var}\left(X\left(t_{1}, t_{2}\right)\right)=$ $\int_{-\infty}^{+\infty} d H_{t_{1}, t_{2}}\left(\omega_{1}, \omega_{2}\right)$. The evolutionary spectral density of the process $\left\{X\left(t_{1}, t_{2}\right)\right\}$ is given by $h_{t_{1}, t_{2}}\left(\omega_{1}, \omega_{2}\right)$ and defined as follows:

$$
h_{t_{1}, t_{2}}\left(\omega_{1}, \omega_{2}\right)=\frac{d H_{t_{1}, t_{2}}\left(\omega_{1}, \omega_{2}\right)}{d \omega_{1} d \omega_{2}}, \quad \omega_{1}, \omega_{2} \in \mathbb{R} .
$$

Assume that the process $\left\{X_{t_{1}, t_{2}}\right\}$ is observed with randomly missing observations. As Bloomfield [2], we consider the process $L_{t_{1}, t_{2}}$ defined as the product of the process $\left\{X_{t_{1}, t_{2}}\right\}$ and an other process $\left\{Y_{t_{1}, t_{2}}\right\}$ defined as follows:

$$
L_{t_{1}, t_{2}}=X_{t_{1}, t_{2}} Y_{t_{1}, t_{2}} \quad \text { where } \quad Y_{t_{1}, t_{2}}=\left\{\begin{array}{ccc}
1 & \text { if } X_{t_{1}, t_{2}} \text { is observed } \\
& 0 & \text { otherwise }
\end{array}\right.
$$


The process $L_{t_{1}, t_{2}}$ is equal to a modified version of the original process $\left\{X_{t_{1}, t_{2}}\right\}$ by replacing the missing observations by $E\left(X_{t_{1}, t_{2}}\right)$ their mean value, which is zero because $\left\{X_{t_{1}, t_{2}}\right\}$ is centred.

To simplify, we suppose, as Bloomfield [2], that $\left\{Y_{t_{1}, t_{2}}\right\}$ is stationary, independent of $X_{t_{1}, t_{2}}$ and satisfying:

$$
\begin{aligned}
& P\left\{Y_{t_{1}, t_{2}}=1\right\}=p>\frac{1}{2}, \\
& P\left\{Y_{t_{1}, t_{2}}=0\right\}=1-p,
\end{aligned}
$$

The assumption of stationarity means that the statistical properties of the process $Y$ does not depend on time. This case is often encountered in practice especially when collecting data provided by devices partially defective. Set

$$
\begin{aligned}
\xi_{r_{1}, r_{2}} & =\frac{1}{p} E\left\{Y_{t_{1}, t_{2}} Y_{t_{1}+r_{1}, t_{2}+r_{2}}\right\} \\
\nu_{q, r, s} & =\frac{1}{p^{2}} E\left\{Y_{t_{1}, t_{2}} Y_{t_{1}+q_{1}, t_{2}+q_{2}} Y_{t_{1}+r_{1}, t_{2}+r_{2}} Y_{t_{1}+s_{1}, t_{2}+s_{2}}\right\} ; \quad q_{i}, r_{i}, s_{i} \in \mathbb{Z}
\end{aligned}
$$

Since $E\left(Y_{t_{1}, t_{2}}\right)=p$, we obtain

$$
\begin{aligned}
\operatorname{Cov}\left\{Y_{t_{1}, t_{2}}, Y_{t_{1}+r_{1}, t_{2}+r_{2}}\right\} & =E\left\{Y_{t_{1}, t_{2}} Y_{+r}\right\}-E\left\{Y_{t_{1}+r_{1}, t_{2}+r_{2}}\right\} E\left\{Y_{t_{1}, t_{2}}\right\} \\
& =p \xi_{r_{1}, r_{2}}-p^{2}=p\left(\xi_{r_{1}, r_{2}}-p\right) .
\end{aligned}
$$

This implies that $\xi_{r_{1}, r_{2}}$ is symmetric in $\left(r_{1}, r_{2}\right)$. In the remainder of this paper, we assume the following hypotheses:

$\left.\mathcal{H}_{1}\right)$ There exists a real number $V>0$ such that

$$
\begin{gathered}
\sum_{q=-\infty}^{\infty}\left|\nu_{r, q, q+s}-\xi_{r_{1}, r_{2}} \xi_{s_{1}, s_{2}}\right| \leq V\left(\left\|\left(r_{1}, r_{2}\right)\right\|+\left\|\left(s_{1}, s_{2}\right)\right\|+1\right)<\infty, \\
\left.\mathcal{H}_{2}\right) \quad \xi>0 \text { and } p \xi_{r_{1}, r_{2}} \geq 2 p-1>0 \quad r_{1}, r_{2} \in \mathbb{Z}
\end{gathered}
$$

Remark 1. - The first hypothesis $\mathcal{H}_{1}$ ) means that the sum,

$$
\sum_{q=-\infty}^{\infty} \operatorname{Cov}\left(Y_{t_{1}, t_{2}} Y_{t_{1}+r_{1}, t_{2}+r_{2}}, Y_{t_{1}+q_{1}, t_{2}+q_{2}} Y_{t_{1}+q_{1}+s_{1}, t_{2}+q_{2}+s_{2}}\right)
$$

is bounded by a function proportional to $p^{2}\left(\left\|\left(r_{1}, r_{2}\right)\right\|+\left\|\left(s_{1}, s_{2}\right)\right\|+1\right)$.

- The second hypothesis $\left.\mathcal{H}_{2}\right)$ implies for each $\left(t_{1}, t_{2}\right)$, the probability that $X_{t_{1}, t_{2}}$ is observed (not missing) is greater than $\frac{1}{2}$.

\section{Estimation of the Evolutionary Spectral Density}

We begin by given some definitions introduced by Priestley ([14], [15]). Let $\mathcal{F}$ the family of oscillatory functions $\left\{A_{t_{1}, t_{2}}\left(\omega_{1}, \omega_{2}\right) e^{i\left(t_{1} \omega_{1}+t_{2} \omega_{2}\right)}\right\}$. For each family $\mathcal{F}$, we 
define the function $\mathcal{B}_{\mathcal{F}}\left(\omega_{1}, \omega_{2}\right)=\int \|\left(\theta_{1}, \theta_{2}\right)||\left|d F_{\omega_{1}, \omega_{2}}\left(\theta_{1}, \theta_{2}\right)\right|$. Let $\mathcal{C}$ in the class of families $\mathcal{F}$ such that $\mathcal{B}_{\mathcal{F}}\left(\omega_{1}, \omega_{2}\right)$ is bounded for all $\left(\omega_{1}, \omega_{2}\right)$. For each family $\mathcal{F}$ we define the following constant $\mathcal{B}_{\mathcal{F}}$ termed the characteristic width of $\mathcal{F}$ :

$$
\mathcal{B}_{\mathcal{F}}=\left[\sup _{\left(\omega_{1}, \omega_{2}\right)} \mathcal{B}_{\mathcal{F}}\left(\omega_{1}, \omega_{2}\right)\right]^{-1}
$$

The characteristic width of the process $X_{t_{1}, t_{2}}$ is defined by $\mathcal{B}_{X}=\sup _{\mathcal{F} \in \mathcal{C}} \mathcal{B}_{\mathcal{F}}$. For more details about definitions see Priestley ([14, , [15]).

In this section, we propose a periodogramm constructed as follows:

$$
I_{t, T}\left(\omega_{01}, \omega_{02}\right)=\left|\sum_{u=t-T}^{t+T} g_{u} \frac{L_{t_{1}-u_{1}, t_{2}-u_{2}}}{S} e^{-i\left(\omega_{01}\left(t_{1}-u_{1}\right)+\left(t_{2}-u_{2}\right) \omega_{02}\right)}\right|^{2}
$$

where $S=\left(2 \pi \sum_{u_{1}, u_{2}} p \xi_{0,0}\left|g_{u_{1}, u_{2}}\right|^{2}\right)^{\frac{1}{2}}$, and $\left\{g_{u_{1}, u_{2}}\right\}$, is a filter satisfying the following conditions:

$C_{1}: g_{u_{1}, u_{2}} \geq 0 ; \quad g_{u_{1}, u_{2}}=g_{-u_{1},-u_{2}}$,

$C_{2}: \sum_{u_{1}, u_{2}, v_{1}, v_{2}} p \xi_{u_{1}-v_{1}, u_{2}-v_{2}} g_{u_{1}, u_{2}} g_{v_{1}, v_{2}}^{*}<\infty$, where $\xi$ is defined in (4)

$C_{3}$ :) $g_{u_{1}, u_{2}}$ has finite "width", defined by:

$$
\mathcal{B}_{g} \simeq \sum_{u_{1}, u_{2}, v_{1}, v_{2}=-\infty}^{+\infty} p\left|\xi_{u_{1}-v_{1}, u_{2}-v_{2}}\right|||\left(u_{1}, u_{2}\right)||\left|g_{u_{1}, u_{2}}\right|\left|g_{v_{1}, v_{2}}^{*}\right|<\infty
$$

$C_{4}: \mathcal{B}_{g}<<\mathcal{B}_{\mathcal{F}}$

$C_{5}$ : For any real numbers $k_{1}, k_{2}$, we have

$\left|\int_{-\infty}^{\infty} \Gamma(s, s) h_{t_{1}, t_{2}}\left(s_{1}+k_{1}, s_{2}+k_{2}\right) d s_{1} d s_{2}-h_{t_{1}, t_{2}}\left(k_{1}, k_{2}\right) \int_{-\infty}^{\infty} \Gamma(s, s) d s_{1} d s_{2}\right|<\frac{\mathcal{B}_{g}}{\mathcal{B}_{\mathcal{F}}}$

where the function $\Gamma$ is defined by:

$$
\Gamma\left(s, s^{\prime}\right)=\sum_{u_{1}, u_{2}, v_{1}, v_{2}} p \xi_{u_{1}-v_{1}, u_{2}-v_{2}} g_{u_{1}, u_{2}} g_{v_{1}, v_{2}}^{*} e^{-i\left(u_{1} s_{1}-v_{1} s_{1}^{\prime}+u_{2} s_{2}-v_{2} s_{2}^{\prime}\right)} .
$$

The function $\Gamma_{1}$ is highly concentred relative to the function $h_{t_{1}, t_{2}}$.

When this condition is satisfied, we say as Priestley (15 page 829) that the function $\Gamma_{1}$ is $\delta$-function with respect to $h_{t_{1}, t_{2}}$ in order $\left(\frac{\mathcal{B}_{g}}{\mathcal{B}_{\mathcal{F}}}\right)$.

$$
C_{6}: g_{u_{1}, u_{2}}=O\left(e^{-\left\|\left(u_{1}, u_{2}\right)\right\|}\right)
$$

The following theorem shows that the periodogram $I_{t, T}\left(\omega_{01}, \omega_{02}\right)$ is an asymptotically unbiased estimator of the evolutionary spectral density $h_{t_{1}, t_{2}}\left(\omega_{01}, \omega_{02}\right)$.

Theorem 1. Let $t_{1}, t_{2}$ be an integer numbers and $\omega_{01}, \omega_{02}$ are real numbers, suppose that $\frac{\mathcal{B}_{g}}{\mathcal{B}_{X}}<\epsilon$, then

$$
E\left[I_{t, T}\left(\omega_{01}, \omega_{02}\right)\right]=h_{t_{1}, t_{2}}\left(\omega_{01}, \omega_{02}\right)+O(\epsilon) .
$$


To prove the theorem 1, we have need the two following lemmas

Lemma 1. For any $t_{1}, t_{2}, t_{1}^{\prime}, t_{2}^{\prime}, \lambda_{1}, \lambda_{2}$ real numbers, we have

$$
\begin{gathered}
\mid \int e^{i\left(t_{1} s 1+t_{2} s_{2}\right)} e^{-i\left(t_{1}^{\prime} s_{1}^{\prime}+t_{2}^{\prime} s_{2}^{\prime}\right.} \Gamma\left(s+k,, s^{\prime}+k^{\prime}\right) d F_{\lambda_{1}, \lambda_{2}}\left(s_{1}, s_{2}\right) d F_{\lambda_{1}, \lambda_{2}}\left(s_{1}^{\prime}, s_{2}^{\prime}\right)- \\
\Gamma\left(k, k^{\prime}\right) \int e^{i\left(t_{1} s_{1}+t_{2} s_{2}\right)} e^{-i\left(t_{1}^{\prime} s_{1}^{\prime}+t_{2}^{\prime} s_{2}^{\prime}\right)} d F_{\lambda_{1}, \lambda_{2}}\left(s_{1}, s_{2}\right) d F_{\lambda_{1}, \lambda_{2}}\left(s_{1}^{\prime}, s_{2}^{\prime}\right) \mid<2 \frac{\mathcal{B}_{g}}{\mathcal{B}_{\mathcal{F}}}
\end{gathered}
$$

Lemma 2. Let $\theta_{1}, \theta_{2}, \lambda_{1}, \lambda_{2}, t_{1}, t_{2}$ and $t_{1}^{\prime}, t_{2}^{\prime}$ be real numbers, we have

$$
\begin{gathered}
\left|A_{t_{1}, t_{2}}\left(\lambda_{1}, \lambda_{2}\right) A_{t_{1}^{\prime}, t_{2}^{\prime}}^{*}\left(\lambda_{1}, \lambda_{2}\right)\right|\left|\Gamma_{t_{1}, t_{2}, t_{1}^{\prime}, t_{2}^{\prime}, \lambda_{1}, \lambda_{2}}\left(\theta_{1}, \theta_{2}\right)-\Gamma(\theta, \theta)\right| \leq 2 \frac{\mathcal{B}_{g}}{\mathcal{B}_{\mathcal{F}}}, \text { where } \\
\Gamma_{t_{1}, t_{2}, s_{1}, s_{2}, \lambda_{1}, \lambda_{2}}\left(\theta_{1}, \theta_{2}\right)=\sum_{u_{1}, u_{2}, v_{1}, v_{2}} p \xi_{u_{1}-v_{1}, u_{2}-v_{2}} g_{u_{1}, u_{2}} g_{v_{1}, v_{2}}^{*} \beta(u, v, \theta)
\end{gathered}
$$

where

$$
\beta(u, v, \theta)=\frac{A_{t_{1}-u_{1}, t_{2}-u_{2}}\left(\lambda_{1}, \lambda_{2}\right) A_{s_{1}-v_{1}, s_{2}-v_{2}}^{*}\left(\lambda_{1}, \lambda_{2}\right)}{A_{t_{1}, t_{2}}\left(\lambda_{1}, \lambda_{2}\right) A_{s_{1}, s_{2}}^{*}\left(\lambda_{1}, \lambda_{2}\right)} e^{-i\left(\left(u_{1}-v_{1}\right) \theta_{1}+\left(u_{2}-v_{2}\right) \theta_{2}\right.} .
$$

In order to obtain a consistent estimate of $\left\{h_{t_{1}, t_{2}}\left(\omega_{01}, \omega_{02}\right)\right\}$, we smooth the periodogram in the neighborhood of the time-instant $\left(t_{1}, t_{2}\right)$ via a weight function:

$$
\widehat{h}_{t_{1}, t_{2}}\left(\omega_{01}, \omega_{02}\right)=\sum_{v_{1}, v_{2} \in M} w_{T_{1}^{\prime}, T_{2}^{\prime}, v_{1}, v_{2}} \widehat{I}_{t_{1}-v_{1}, t_{2}-v_{2}}\left(\omega_{01}, \omega_{02}\right) .
$$

where $w_{T_{1}^{\prime}, T_{2}^{\prime}, v_{1}, v_{2}}$ is a weight-function depending on the parameters $T_{1}^{\prime}, T_{2}^{\prime}$ and satisfying

a) $w_{T_{1}^{\prime}, T_{2}^{\prime}, v_{1}, v_{2}} \geq 0$, for all $v_{1}, v_{2}, T_{1}^{\prime}, T_{2}^{\prime}$

b) $w_{T_{1}^{\prime}, T_{2}^{\prime}, v_{1}, v_{2}}=0, v_{1}, v_{2} \notin M$, where $M$ is a set of integers surrounding zero.

c) $w_{T_{1}^{\prime}, T_{2}^{\prime}, v_{1}, v_{2}}=w_{T_{1}^{\prime}, T_{2}^{\prime},-v_{1},-v_{2}}$,

d) $\sum_{v_{1}, v_{2} \in M} w_{T_{1}^{\prime}, T_{2}^{\prime}, v_{1}, v_{2}}=1$,

e) $\sum_{v_{1}, v_{2} \in M} w_{T_{1}^{\prime}, T_{2}^{\prime}, v_{1}, v_{2}}^{2}<\infty$.

f) We assume that there exists a constant $C$ such that

$$
\begin{aligned}
\lim _{T_{1}^{\prime}, T_{2}^{\prime} \rightarrow \infty} & T_{1}^{\prime}, T_{2}^{\prime} \sum_{u_{1}, u_{2} \in M}\left|W_{T_{1}^{\prime}, T_{2}^{\prime}, u_{1}, u_{2}}\right|^{2}=C, \text { where } \\
W_{T_{1}^{\prime}, T_{2}^{\prime}, u_{1}, u_{2}}= & \sum_{v_{1}, v_{2} \in M} e^{-i\left(u_{1} v_{1}+u_{2} v_{2}\right)} w_{T_{1}^{\prime}, T_{2}^{\prime}, v_{1}, v_{2}} .
\end{aligned}
$$

The following theorem show that the estimator $\widehat{h}_{t_{1}, t_{2}}\left(\omega_{01}, \omega_{02}\right)$ is an asymptotically unbiased of the (weighted) average value of $h_{t_{1}, t_{2}}\left(\omega_{01}, \omega_{02}\right)$ in the neighborhood of $\left(t_{1}, t_{2}\right)$. 
Theorem 2. Let $-\pi \leq \omega_{01}, \omega_{02} \leq \pi$, suppose that $\frac{\mathcal{B}_{g}}{\mathcal{B}_{X}}<\epsilon$, then

$$
E\left[\widehat{h}_{t_{1}, t_{2}}\left(\omega_{01}, \omega_{02}\right)\right]=\bar{h}_{t_{1}, t_{2}}\left(\omega_{01}, \omega_{02}\right)+O(\epsilon)
$$

where $\bar{h}_{t_{1}, t_{2}}\left(\omega_{01}, \omega_{02}=\sum_{v_{1}, v_{2} \in M} w_{T_{1}^{\prime}, T_{2}^{\prime}, v_{1}, v_{2}} h_{t_{1}-v_{1}, t_{2}-v 2}\left(\omega_{01}, \omega_{02}\right)\right.$

To show that the variance converges to zero, as Priestley ([14]) and Mélard [10], we assume that the process $L_{t_{1}, t_{2}}$ is Gaussian.

Theorem 3. Let $-\pi \leq \omega_{1}, \omega_{2} \leq \pi$ and suppose that the process $L_{t_{1}, t_{2}}$ is Gaussian, then we have

$$
\operatorname{Var}\left[\widehat{h}_{t_{1}, t_{2}}\left(\omega_{01}, \omega_{02}\right)\right]=O\left(\frac{1}{T_{1}^{\prime}, T_{2}^{\prime}}\right) .
$$

\section{Numerical Studies}

As in Bloomfield [2], we suppose that our process $\left\{X_{t, s}\right\}_{t, s \in \mathbb{Z}}$ is observed at the successively instants $\left(t_{1}, s_{1}\right),\left(t_{2}, s_{2}\right), \ldots,\left(t_{n}, s_{n}\right)$ where $\tau_{i}=\left|t_{i+1}-t_{i}\right| \tau_{i}^{\prime}=\mid s_{i+1}-$ $s_{i} \mid$ are independent random variables, each with the probability distribution $\left\{f_{r_{1}, r_{2}}=P\left[\left(\tau, \tau^{\prime}\right)=\left(r_{1}, r_{2}\right)\right]\right\}$, and finite mean $p^{-1}$. As in Feller ([5], pp 282$283)$, we define a process $\left\{Y_{t, s}^{\prime}\right\}$ which coincides with $\left\{Y_{t, s}\right\}$ except at origin $Y_{0,0}^{\prime}=1$. the event " $Y^{\prime}=1$ " is termed persistent and recurrent event. Using (6) we obtain

$$
\begin{aligned}
\xi_{r_{1}, r_{2}} & =p^{-1} E\left\{Y_{t_{1}, t_{2}} Y_{t_{1}+r_{1}, t_{2}+r_{2}}\right\}=P\left\{Y_{t_{1}+r_{1}, t_{2}+r_{2}}=1 / Y_{t_{1}, t_{2}}=1\right\} \\
& =P\left\{Y_{r_{1}, r_{2}}^{\prime}=1\right\}
\end{aligned}
$$

Feller ([5], pp 282-283) has shown that

$$
\xi_{r_{1}, r_{2}}=\sum_{s=1}^{r_{1}, r_{2}} f_{s_{1}, s_{2}} \xi_{r_{1}-s_{1}, r_{2}-s_{2}}, r_{i}, s_{i}=1,2 \ldots
$$

The processus $L_{t_{1}, t_{2}}$ was obtained from $X_{t, s}$ by omitting certain observations with a renewal-type mechanism defined above with $f_{1,1}=\frac{8}{9}, f_{2,2}=\frac{1}{9}, f_{r_{1}, r_{2}}=0$ otherwise.

The simulation of the process $X$ :

Using the same method in [1] for the simulation of Markov Gauss random field, we simulate the Gaussian random field $Y=\left\{Y\left(n_{1}, n_{2}\right)\right\}_{n_{1}, n_{2} \in \mathbb{Z}}$ such that $R_{Y}\left(n_{1}, n_{2}\right)$ the covariance function is given by $R_{Y}\left(n_{1}, n_{2}\right)=\mathrm{e}^{-\sqrt{\left(n_{1}+n_{2}\right)}}$, and its spectral density is $f_{Y}\left(\lambda_{1}, \lambda_{2}\right)=\frac{1}{\pi\left(1+\lambda_{1}^{2}+\lambda_{2}^{2}\right)}$.

the random field $X_{t, s}, t, s \in \mathbb{Z}$ is given by the following model

$$
X_{t, s}=c_{t, s} Y_{t, s}, \quad t, s \in \mathbb{Z} .
$$


where $c_{t, s}=e^{-\frac{(t+s-500)^{2}}{2 * 200^{2}}} A_{t, s}\left(\omega_{1}, \omega_{2}\right)=c_{t, s}$ is independent of $\omega$. With respect to the family $\mathcal{F}=\left\{c_{t, s} e^{i\left(\omega_{1} t+\omega_{2} s\right.}\right\}, X(t, s)$ has evolutionary spectral density function $h_{t_{1}, t_{2}}\left(\omega_{1}, \omega_{2}\right)=c_{t_{1}, t_{2}}^{2} f_{Y}\left(\omega_{1}, \omega_{2}\right)$.

The curve of the estimator with 5000 observations (Fig. 2) and that of the spectral density (Fig. 1) are very similar. So the estimator is quite satisfactory. If we take more observations (around 10000), the estimator becomes more smoother and the curve approaches the density much.

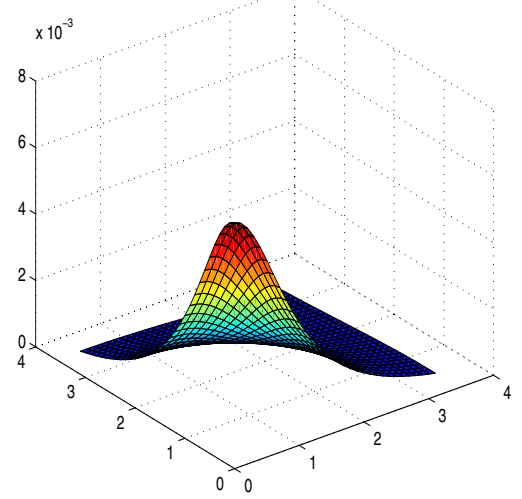

Fig. 1. Density $h_{100,12}$

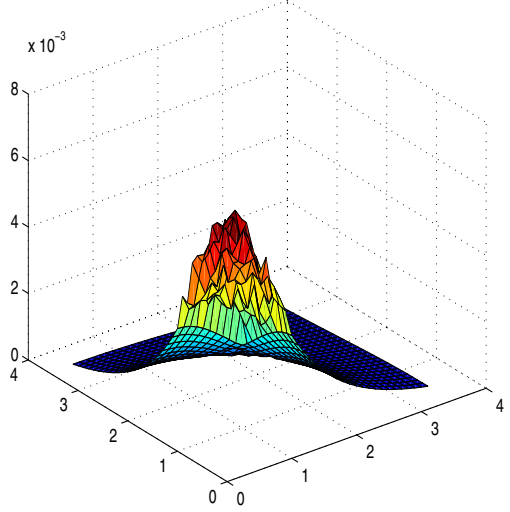

Fig.2. Estimator $\widehat{h}_{100,12}$

\section{Conclusion}

We have proposed in this paper some results about the estimation of the evolutionary spectral density for non-stationary random fields where the data observed are collected with missing values. The approach is based on the technique used by Bloomfield [2] for stationary processes combining estimates of evolutionary spectrum introduced by Priestley (14]). This work could be applied to several cases when the process is non-stationary as for example for:

- the segmentation of a sequence of images of a dynamic scene, detecting weeds in a farm field.

- the study of geostatistical mapping of certain chemical factors in agricultural soil.

This work could be supplemented by the study of optimal smoothing parameters using cross validation methods that have proven in the field. It will also be extended to non-Gaussian process by assuming some hypotheses as for example the cumulants are finite.

Acknowledgments. I would like to thank the anonymous referees for their interest in this paper and their valuable comments and suggestions. 


\section{References}

1. Al-Shoshan, A.I., Chaparro, L.F.: Identification of non-minimum phase systems using evolutionary spectral theory. Signal Processing 55(1), 79-92 (1996)

2. Bloomfield, P.: Spectral analysis with randomly missing observations. J. Roy. Statistical Society 32(3), 369-380 (1970)

3. Cabrera, S.D., Flores, B.C., Thomas, G., Vega-Pineda, J.: Evolutionary spectral estimation based on adaptive use of weighted norms. In: Luk, F.T. (ed.) Advanced Signal Processing Algorithms, Architectures, and Implementations IV. SPIE, vol. 2027, pp. 168-179 (1993)

4. Caetano, M., Rodet, X.: Evolutionary spectral envelope morphing by spectral shape descriptors. In: Proceeding of International Computer Music Conferences (ICMC) (August 2009)

5. Feller, W.: An Introduction to Probability Theory and its Applications, 2nd edn., vol. I. Wiley, New York (1957)

6. Jones, R.H.: Spectrum estimation with missing observations. Annals of the Institute of Statistical Mathematics 23(1), 387-398 (1971)

7. Hung, J.-C.: A genetic algorithm approach to the spectral estimation of time series with noise and missed observations. Information Sciences 178(24), 4632-4643 (2008)

8. Kayhan, A.S., El-Jaroudi, A., Chaparro, L.F.: Data-Adaptive Evolutionary Spectral Estimation. IEEE Transactions on Signal Processing 43(1), 204-213 (1995)

9. Kayhan, A.S., Amine, M.G.: Spatial evolutionary spectrum for DOA estimation and blind signal separation. IEEE Transactions on Signal Processing 48(3), 791-798 (2000)

10. Mélard, G., de Schutter, A.H.: Contributions to evolutionary spectral theory. Journal of Time Series Analysis 10(1), 41-63 (1988)

11. Messaci, F.: Estimation de la densité spectrale d'un processus en temps continu par échantillonnage poissonnien, Univ de Rouen (Thèse de 3 ème cycle) (1986)

12. Parzen, E.: On spectral analysis with missing observations and amplitude modulation. Sankhya, Series A 25, 383-392 (1963)

13. Broersen, P.M.T.: Automatic spectral analysis with missing data. Digital Signal Processing 16(6), 754-766 (2006)

14. Priestley, M.B.: Evolutionary spectra and non-stationary processes. J. Roy. Statist. Soc. Ser., B 27, 204-237 (1965)

15. Priestley, M.B.: Spectral analysis and time series. Probability and Mathematical Statistics. Academic Press (1981)

16. Rachdi, M., Sabre, R.: Mixed-spectra analysis for stationary random fields. Statistical Methods and Applications 18, 333-358 (2009)

17. Sabre, R.: Spectral density estimation for stationary stable random fields. Journal Applications Mathematicae 23(2), 107-133 (1995)

18. Sabre, R.: Discrete estimation of spectral density for symmetric stable process. Statistica 2, 1-26 (2000)

19. Shah, S.I., Chaparro, L.F., El-Jaroudi, A., Furman, J.M.: Evolutionary Maximum Entropy Spectral Estimation and HeartRate Variability Analysis. Multidimensional Systems and Signal Processing 9(4), 453-458 (1998)

20. Tezcan, J.: Evolutionary Power Spectrum Estimation Using Harmonic Wavelets. Seismic Design and Analysis of Structures, 37-41 (2003)

21. Wang, Y., Stoica, P., Li, J., Marzetta, T.L.: Nonparametric spectral analysis with missing data via the EM algorithm. Digital Signal Processing 15(2) (2005) 